\title{
Pseudopentaceros richardsoni (Osteichthyes: Pentacerotidae) en el Océano Pacífico suroriental: morfometría, parásitos y comentarios sobre la familia en el área
}

\author{
Pseudopentaceros richardsoni (Osteichthyes: Pentacerotidae) in the southeastern \\ Pacific Ocean: morphometry, parasites and comments about the family in the area
}

\section{Germán Pequeño ${ }^{1}$, Verónica Valdebenito² y Gabriela Muñoz ${ }^{3}$}

\author{
${ }^{1}$ Instituto de Zoología ‘Ernst F. Kilian’, Universidad Austral de Chile, Casilla 567, Valdivia, Chile. gpequeno@uach.cl \\ ${ }^{2}$ Instituto de Fomento Pesquero, Av. Colón 3656, Talcahuano, Chile \\ ${ }^{3}$ Facultad de Ciencias del Mar y de Recursos Naturales, Universidad de Valparaíso, Casilla 5080, Reñaca, Viña del Mar, Chile
}

\begin{abstract}
This study communicates a new record of six specimens of Pseudopentaceros richardsoni, collected from $500 \mathrm{Nm}$ off Coronel, Chile. In addition, this study provides: the morphometry of this species, a general view of the geographical distribution of the family Pentacerotidae for the southeastern Pacific Ocean, and information on the metazoan parasites found in the specimens collected. The fish body length ranged from $275-390 \mathrm{~mm}$. Three parasite species were determined, Rhadynorhynchus trachuri, Lampritrema sp. and Hysterothylacium sp. This is the third record of P. richardsoni for Chilean waters and the first record of its parasites for the southeastern Pacific Ocean.
\end{abstract}

Key words: Ichthyogeography, armourhead fish, morphometry

\section{INTRODUCCIÓN}

La primera especie de la familia Pentacerotidae encontrada en aguas chilenas se conoció como Pentaceros knerii Steindachner 1866 (Fowler 1943), hoy reconocida como sinónimo júnior de Pseudopentaceros richardsoni (Smith, 1844), según Eschmeyer $\left(2010^{1}\right)$. La descripción de Steindachner (1866) se hizo sobre la base de ejemplares capturados frente al Cabo de Hornos, un hito geográfico que presenta especial interés biogeográfico dada sus particulares características costeras. Probablemente, dada la localidad tipo de la especie descrita por Steindachner, fue considerada como especie propia de los canales patagónicos (Mann 1954), sin embargo su distribución es más amplia. Esta especie es característica del hemisferio sur, donde ha sido registrada en varias localidades (Borets 1980, Humphreys \& Tagami 1986).

Pequeño $(1989,1997)$ ha brindado alguna información sobre otras especies de la familia Pentacerotidae para Chile. De estas publicaciones se desprende que varios registros son de muestras esporádicas, señalando a cinco especies: Pentaceros capensis Cuvier, 1829; P. decacanthus Günther, 1859; P. japonicus Steindachner, 1883; P. quinquespinis Parin \& Kotlyar, 1988 y Pseudopentaceros richardsoni.

La familia Pentacerotidae es poco conocida, aunque hay algunos estudios biológicos realizados en diferentes zonas (e.g., Hardy 1983, Heemstra 1986, Mundy \& Moser 1997). En Chile, además de la presencia de Pseudopentaceros richardsoni en el extremo austral del país, sólo Pentaceros capensis ha sido encontrada en la costa de Chile continental, pues las otras tres especies han sido registradas en Isla de Pascua (Randall \& Cea 2010) y en la cordillera sumergida de Salas y Gómez (Parin et al 1980, Parin \& Kotlyar 1988). Pseudopentaceros richardsoni también integra la ictiofauna de Argentina (Menni et al. 1984).

La mayoría de los ejemplares de la familia Pentacerotidae registrados en Chile, no han sido depositados en colecciones chilenas, por lo que cualquier comparación o verificación de la identidad específica de estos ejemplares resulta difícil. Es así, que el presente estudio tuvo como 
objetivos, comunicar este nuevo hallazgo frente a Chile, complementar las observaciones comunicadas anteriormente, tales como las de Fowler (1943), Steindachner (1866) y Parin \& Kotlyar (1988), y aportar con antecedentes parasitológicos, que son escasos a nivel mundial.

\section{MATERIALES Y MÉTODOS}

Se capturaron seis ejemplares de Pseudopentaceros richardsoni con red de cerco, como parte de la fauna incidental o acompañante de las pesquerías del jurel Trachurus murphyi Nichols, 1920, alrededor de 500 millas náuticas al SW de Coronel ( $37^{\circ} 00^{\prime} \mathrm{S}, 73^{\circ} 10^{\prime} \mathrm{O}$ ), Chile, en el mes de julio de 2010, a una profundidad de 70 a $90 \mathrm{~m}$.

Los ejemplares fueron congelados para su traslado. Tres ejemplares fueron utilizados en el análisis morfométrico, medidos con un pié de metro Mitutoyo digital de $1 \mathrm{~mm}$ de precisión y fijados inicialmente en formalina al $10 \%$, y luego en etanol al $70 \%$. Las medidas morfométricas consideradas fueron: LT, longitud total; LE, longitud estándar; LC, longitud de la cabeza; AMC, Altura máxima del cuerpo; DPD, distancia pre-dorsal; DPA, distancia pre-anal; AMPC, altura mínima del pedúnculo caudal; DO, diámetro ocular; LBD, longitud de la base de la aleta dorsal; LBA, longitud base aleta anal; LP, longitud aleta pectoral; LPE, longitud aleta pélvica o ventral; M+P, longitud maxilar + premaxilar. Los tres ejemplares preservados, fueron depositados en el Museo Nacional de Historia Natural de Chile, con los números de catálogo MNHNCL-P-6892, 6893 y 6894.

Todos los ejemplares fueron examinados por parásitos, lo que consistió en revisar externa e internamente el cuerpo de cada pez a ojo desnudo. La disección y tamizado (con malla de $250 \mu \mathrm{m}$ ) de los órganos fueron necesarios para recolectar los parásitos, que luego fueron fijados en formalina al $10 \%$. Posteriormente, algunos helmintos fueron teñidos con hematoxilina, deshidratados con una serie de alcoholes de $70^{\circ}$ hasta $100^{\circ}$ y luego montados en bálsamo de Canadá, para proceder a su observación y determinación taxonómica mediante literatura especializada (Cannon 1977, Gibson 2002, Amin et al. 2011). Para cada taxón de parásito se calculó la prevalencia (PREV: porcentaje de hospedadores parasitados) y la abundancia promedio (ABU: número promedio de especímenes parásitos por individuo hospedador examinado \pm desviación estándar). Uno a cuatro ejemplares de cada taxón parasitario fue depositado en el Museo de Zoología de la Universidad de Concepción, catalogados desde el número MZUC 29826 al MZUC 29829.

\section{RESUltados Y DiscusióN}

La longitud total de los seis ejemplares (tres hembras y tres machos) fluctuó entre 27,5 y 39,0 cm y su peso corporal (incluidas las vísceras) osciló entre 453,0 y 988,7g. Todos los individuos presentaron las cualidades morfológicas y morfométricas propias de Pseudopentaceros richardsoni (Tabla 1).

\section{MORFOMETRÍA}

Altura del cuerpo entre 2,3 y 2,4 veces en la LE. Cuerpo comprimido, ovalado. Cabeza comprimida, de extremo anterior romo. LC alrededor de 3,5 veces la LE. Boca más bien pequeña, terminal, dirigida ligeramente hacia arriba; la mandíbula superior no alcanza el nivel del borde anterior del ojo; mandíbula inferior ligeramente prognata. Nariz suavemente bulbosa. Dientes finos, cónicos, en bandas o parches sobre ambas mandíbulas y el vómer. Espacio interorbital amplio, elevado sobre las órbitas pero plano, contenido menos de tres veces en la LC. Ojo alrededor de

Tabla 1. Principales mediciones merísticas y morfométricas de tres ejemplares de Pseudopentaceros richardsoni / Main morphometric and meristic measurements for three specimens of Pseudopentaceros richardsoni

\begin{tabular}{lrcr}
\hline Características & \multicolumn{3}{c}{ Ejemplares } \\
& 1 & 2 & 3 \\
\hline Longitud total (mm) & 365 & 344 & 275 \\
Longitud estándar (mm) & 307 & 290 & 275 \\
En \% de la LE: & & & \\
Longitud de la cabeza & 28,0 & 29,3 & 29,8 \\
Maxilar + premaxilar & 8,5 & 8,3 & 8,7 \\
Diámetro ocular & 6,8 & 7,2 & 7,6 \\
Distancia predorsal & 37,8 & 40,0 & 35,6 \\
Distancia preanal & 63,8 & 64,1 & 68,0 \\
Altura máxima del cuerpo & 40,7 & 37,2 & 41,1 \\
Altura mínima pedúnculo caudal & 10,4 & 10,3 & 10,9 \\
Longitud base aleta dorsal & 54,4 & 54,8 & 56,0 \\
Longitud base aleta anal & 50,1 & 51,4 & 52,7 \\
Longitud aleta pectoral & 21,8 & 22,8 & 22,5 \\
Longitud aleta pélvica & 22,8 & 22,1 & 22,2 \\
$\mathrm{~N}^{\circ}$ branquispinas brazo inferior & 13 & 14 & 13 \\
$\mathrm{~N}^{\circ}$ branquispinas brazo superior & 5 & 6 & 6 \\
$\mathrm{~N}^{\circ}$ espinas aleta dorsal & 14 & 14 & 14 \\
$\mathrm{~N}^{\circ}$ radios aleta dorsal & 9 & 10 & 9 \\
$\mathrm{~N}^{\circ}$ espinas aleta anal & 4 & 4 & 4 \\
$\mathrm{~N}^{\circ}$ radios aleta anal & 9 & 9 & 8 \\
$\mathrm{~N}^{\circ}$ radios aleta pectoral & 17 & 17 & 17 \\
\hline & & &
\end{tabular}


cuatro veces la LC. Opérculos, preopérculos y subopérculos, cleitros, nasales, frontales y otros huesos del cráneo, marcados por fuertes estrías radiales. Pedúnculo caudal comprimido, su altura contenida entre 9 y 11 veces en la LE. Longitud de la base de la aleta dorsal completa, mayor que la mitad de la LE. Aletas pélvicas y pectorales de similar longitud (Fig. 1A y 1B).

La descripción de los especímenes de este estudio concuerda con aquella entregada por Hart (1973), con algunas diferencias menores, e.g., el pedúnculo caudal es ligeramente más alto y las aletas pectorales más cortas en los ejemplares aquí estudiados.

El hallazgo de estos ejemplares en mar abierto, entre el Cabo de Hornos y la cordillera sumergida de Salas y Gómez, frente a la zona centro-sur de Chile constituye el tercer registro de $P$. richardsoni. Al mismo tiempo, permite observar una continuidad en la distribución geográfica de la especie, ya que en la costa atlántica de América del Sur, también hay antecedentes de su presencia en las cercanías de la ciudad de Mar del Plata, Argentina (Menni et al. 1984). Además, este pez habita aguas de Sudáfrica,

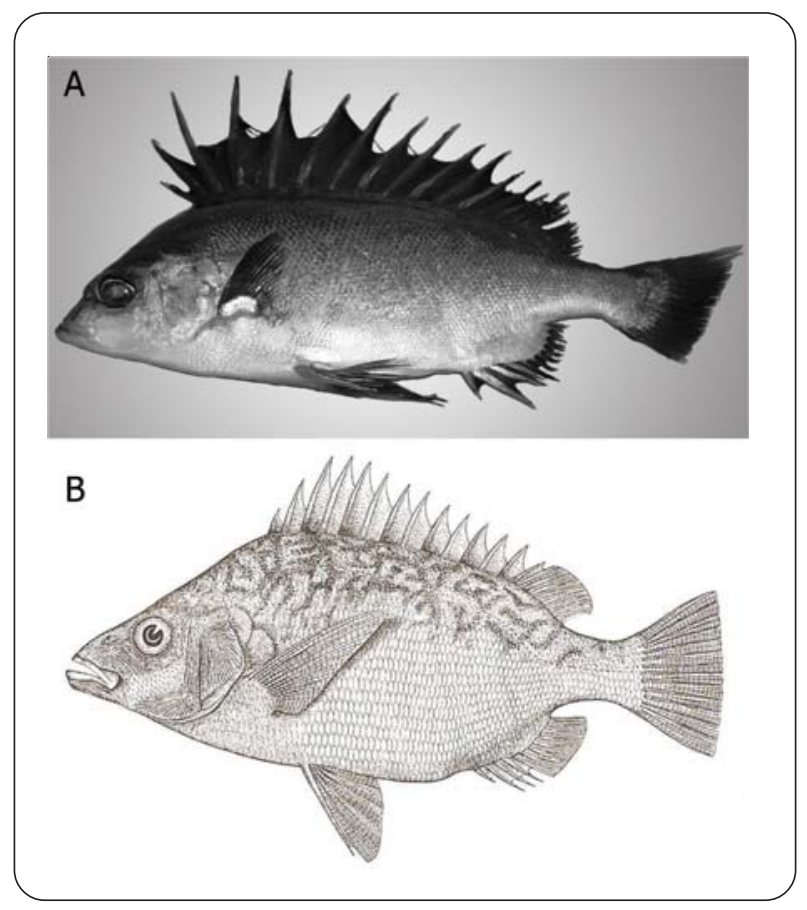

Figura 1. A) Ejemplar de Pseudopentaceros richardsoni de $344 \mathrm{~mm}$ de LT (presente estudio, y B) Pseudopentaceros richardsoni (tomado y modificado de Hart, 1973) / A) Specimen of Pseudopentaceros richardsoni, $344 \mathrm{~mm}$ total length (from this study, and B) Pseudopentaceros richardsoni (slightly modified from Hart, 1973)
Australia y Nueva Zelanda (Hardy 1983, Heemstra 1986), por lo que se la considera de distribución circumglobal en el hemisferio sur (Humphreys \& Tagami 1986), permitiendo con el presente reporte cubrir las áreas donde su presencia sólo se presumía. Otra especie de la misma familia, Pentaceros capensis, fue registrada en una costa rocosa de Aysén (Pequeño et al. 1992), siendo también conocida en aguas de Sudáfrica, islas Reunión (Francia) y posiblemente Australia (Eschmeyer 2010¹). Otras especies de la familia, han sido descubiertas y registradas en el mar de Chile, tanto en islas oceánicas, como en cordilleras sumergidas (Parín et al. 1980, Parin \& Kotlyar 1988, Pequeño et. al. 2003, Randall \& Cea 2010), lo cual permite esbozar un esquema general de la distribución de la familia en el océano Pacífico suroriental (Fig. 2). En Isla de Pascua se encuentra Pentaceros decacanthus, considerada entre la ictiofauna costera (Randall \& Cea

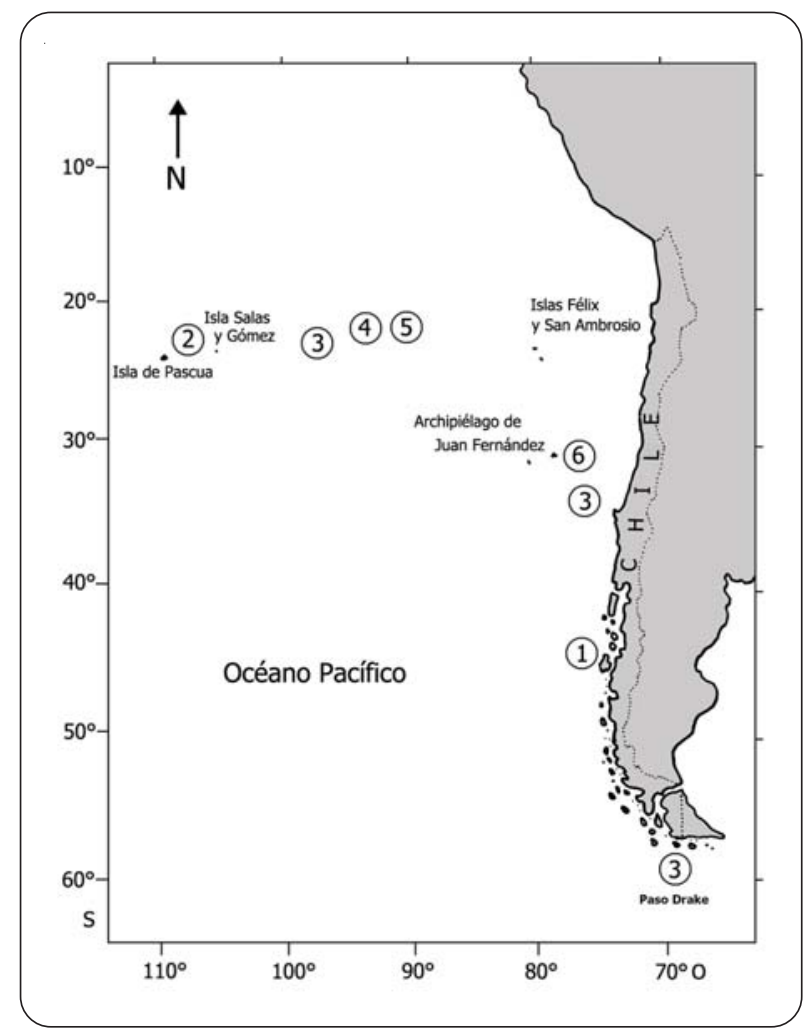

Figura 2. Lugares de registros de peces de la familia Pentacerotidae en el Océano Pacífico suroriental, frente a Chile: 1) Pseudopentaceros capensis, 2) Pentaceros decacanthus, 3) P. richardsoni, 4) P. japonicus, 5) P. quinquespinis y 6) Pseudopentaceros sp. / Locations of geographical records for fishes of the family Pentacerotidae in the eastern Pacific Ocean off the coast of Chile: 1) Pseudopentaceros capensis, 2) Pentaceros decacanthus, 3) P. richardsoni, 4) P. japonicus, 5) P. quinquespinis and 6) Pseudopentaceros sp. 
2010), especie que también habita en aguas del Pacífico suroccidental, en la parte sur de Australia y en Nueva Zelandia (Paulin et al. 1989). Pentaceros japonicus ha sido registrada en los montes submarinos de Nazca y de Salas y Gómez (Parin et al. 1980), también habita en aguas de Japón (islas Ogasawara), en las crestas de Hawaii, en Nueva Caledonia y sur de Corea (Lindberg \& Krasyukova 1969, Eschmeyer 2010¹). Pentaceros quinquespinis es conocida en el monte Bol'shaya, en la cordillera sumergida

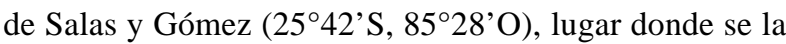
descubrió originalmente. Queda por dilucidar si ejemplares pentacerótidos no identificados registrados en los montes sumergidos de Juan Fernández (Pequeño et al. 2003) pertenecen a alguna de las especies señaladas, o si se trata de una especie aún no descrita.

Pseudopentaceros richardsoni y Pentaceros capensis son habitantes de la línea de costa continental de Chile, por lo tanto, es conveniente conocer las diferencias morfológicas básicas entre adultos de ambas especies, para los cual se propone la siguiente clave:

Aleta dorsal con XII-XIII espinas y línea lateral con alrededor de 50 escamas. Radio más corto de la aleta pectoral alcanza el nivel de la base de la primera espina de la aleta anal......................................... Pentaceros capensis Aleta dorsal con XIV-XV espinas y línea lateral con 74 a 86 escamas. Radio más corto de la aleta pectoral no alcanza el nivel de la base de la primera espina de la aleta anal...............................Pseudopentaceros richardsoni

La información morfológica obtenida de fotografías y dibujos de $P$. richardsoni, de distintas fuentes, muestra algunas diferencias aunque no a nivel merístico (e.g., Follett \& Dempster 1963, Zama et al. 1977, Ayling \& Cox 1982, Heemstra 1986, Humphreys \& Tagami 1986). Las observaciones documentadas de la especie se han basado en ejemplares de diferentes tamaños, sexos y zonas geográficas, variables que pueden influir en la morfología de los especímenes, por lo tanto en este estudio la especie fue referida en términos de sensu lato.

De todos modos, la familia Pentacerotidae requiere un estudio conjunto en Chile, para reunir el material existente $y$, eventualmente pesquisar la existencia de ejemplares en colecciones aún no catalogados, tanto en Chile como en el extranjero, tareas para desarrollar a futuro.

\section{Parásitos}

Cinco de los seis ejemplares examinados estaban parasitados. Se recolectaron en total 36 endoparásitos, encontrados desde la tercera porción del intestino. Se determinaron tres taxa: un nemátodo al estado larval, Hysterothylacium sp. (Anisakidae) (PREV: 17\%, ABU: 0,2 \pm 0,4); un digeneo adulto Lampritrema sp. (Lampritremidae) (PREV: 17\%, ABU: 0,2 $\pm 0,4$ ) que posiblemente sea $L$. miescheri que es la única especie del género, sin embargo algunas características reproductivas de la especie no fueron claramente observadas en el único ejemplar obtenido; y 34 acantocéfalos Rhadinorhynchus trachuri (Rhadinorhynchidae) (PREV: 83\%, ABU: 5,7 \pm $6,9)$. No se encontró ningún ectoparásito ni parásito en la musculatura (en 3 ejemplares revisados).

En general, a nivel mundial existe escaso conocimiento de los parásitos en la familia Pentacerotidae (e.g., Humphreys \& Tagami 1986, Humphreys et al. 1993, Machida \& Araki 2002). Para Pseudopentaceros richardsoni sólo existe un registro, el cestodo larval Gymnorhynchus gigas hospedado en la musculatura (Alioshkina et al. 1985 fide Humphreys \& Tagami 1986), mientras que en Chile no hay información al respecto (ver Muñoz \& Olmos 2008).

Los tres taxa parasitarios encontrados son comunes en peces pelágicos; Lampritrema miescheri ha sido registrada en peces de los géneros Lampris, Brama, Thyrsites y Trachipterus (Gibson 2002, Oliva 1984), mientras que Rhadinorhynchus trachuri ha sido registrado principalmente en peces de las familias Scombridae y Carangidae en las costas sudamericanas, incluyendo Chile (Rego 1987, Santos et al. 2008, Muñoz \& Olmos 2008). Por su parte, Hysterothylacium es un parásito común en varias especies de peces bentodemersales, pelágicos y de profundidad (Muñoz \& Olmos 2008). Por lo tanto, los registros de parásitos son nuevos para la especie $P$. richardsoni.

Debido a que los estómagos de los ejemplares examinados se encontraron vacíos no fue posible indagar sobre las presas que puedan transmitir estos parásitos a Pseudopentaceros richardsoni. Se ha descrito que los peces pentacerótidos se alimentan de zooplancton, principalmente copépodos y anfípodos (Humphreys \& Tagami 1986), los cuales en el sistema pelágico participan en los ciclos de vida de varias especies de parásitos, actuando como hospedadores intermediarios (Marcogliese 1995).

El escaso conocimiento biológico sobre Pseudopentaceros richardsoni, especialmente en el Pacífico suroriental, se debe por el difícil acceso de muestreo de los especímenes, dada su distribución 
pelágico-oceánica para juveniles y de mayor profundidad para adultos. En todo caso, la baja riqueza de parásitos es el resultado acorde al relativamente escaso número de ejemplares examinados. Sin embargo, hay que señalar que los peces pelágico-oceánicos suelen tener menor abundancia de ectoparásitos, posiblemente porque los estadios parasíticos libres de distintas especies parásitas tienen corta vida activa y limitada distribución, lo que contribuye a que la transmisión de parásitos entre hospedadores sea más difícil.

Básicamente, los peces pentacerótidos cuentan con escasos antecedentes biológicos y se capturan solo en algunas localidades, e.g., Japón, con una distribución más cercana a la costa y son capturados comercialmente, sin embargo, en Chile, las capturas de estos peces son incidentales y forman parte de la fauna acompañante de peces pelágicos, lo que dificulta estudios basados en tamaños muestrales mayores o muestreos frecuentes en el tiempo.

\section{Agradecimientos}

Se agradece el aporte de los ejemplares estudiados al Instituto de Fomento Pesquero de Chile (IFOP). Importante apoyo bibliográfico fue dado por Richard L. Haedrich (Memorial University, Newfoundland, Canadá) y M. Eric Anderson (Grahamstown, South Africa). Valeria Pérez (Instituto de Zoología, UACH) prestó valioso apoyo en el trabajo fotográfico. Este estudio recibió apoyo parcial del Proyecto DID-UACH-2005-03.

\section{LITERATURA CITADA}

Amin OM, RA Heckmann \& Ng Van Ha. 2011. Description of two new species of Rhadinorhynchus (Acanthocephala, Rhadinorhynchidae) from marine fish in Halong Bay, Vietnam, with a key to species. Acta Parasitologica 56(1): 67-77.

Ayling T \& G Cox. 1982. Sea Fishes of New Zealand, 343 pp. W. Collins Publishers, Auckland.

Borets LA. 1980. The distribution and structure of the range of the boarfish Pentaceros richardsoni. Journal of Ichthyology 20(3): 141-143.

Cannon LRG. 1977. Some larval ascaridoids from south-eastern Queensland marine fishes. International Journal for Parasitology 7: 233-243.

Follett WI \& LJ Dempster. 1963. Relationships of the percoid fish Pentaceros richardsoni Smith, with description of a specimen from the coast of California. Proceedings of the California Academy of Sciences 32: 315-338.
Fowler HW. 1943. Fishes of Chile. Systematic catalog. Revista Chilena de Historia Natural 41-42: 15-116; 275-350.

Gibson DI. 2002. Family Hirudinellidae Dollfus 1932. En: Gibson DI, A Jones \& RA Bray (eds). Keys to the Trematoda, pp. 375-378. CABI Publishing, Wallingford.

Hardy GS. 1983. A revision of the fishes of the family Pentacerotidae (Perciformes). New Zealand Journal of Zoology 10: 177-220.

Hart JL. 1973. Pacific fishes of Canada. Bulletin, Fisheries Research Board of Canada 180: 1-740.

Heemstra PC. 1986. Family $N^{\circ} 203$ Pentacerotidae. En: Smith MM \& PC Heemstra (eds). Smith’s Sea Fishes, pp. 622623. Macmillan South Africa, Johannesburg.

Humphreys RL Jr \& DT Tagami. 1986. Review and current status of research on the biology and ecology of the genus Pseudopentaceros. En: Uchida RN, S Hayasi \& GW Boehlert (eds). Environment and resources of seamounts in the North Pacific, NOAA Technical Report NMFS 43: 55-62.

Humphreys RL Jr, MA Crossler \& CM Rowland. 1993. Use of a monogenean gill parasite and feasibility of condition indices for identifying new recruits to a seamount population of armorhead Pseudopentaceros wheeleri (Pentacerotidae). Fishery Bulletin 91: 455-463.

Lindberg GU \& ZV Krasyukova. 1969. Fishes of the Sea of Japan and the adjacent areas of the Sea of Okhotsk and the Yellow Sea. Part 3, Teleostomi, XXIX Perciformes, 498 pp. Akademiya Nauk SSSR, Leningrad. [Israel Program for Scientific Translations, Jerusalem 1971].

Machida M \& J Araki. 2002. Three new species of digenean trematodes found in deep-sea fishes of Japan and adjacent waters. Bulletin of the National Science Museum, Tokyo Series A, Zoology 24: 195-200.

Mann G. 1954. La vida de los peces en aguas chilenas, 343 pp. Instituto de Investigaciones Veterinarias y Universidad de Chile, Santiago.

Marcogliese DJ. 1995. The role of zooplankton in the transmission of helminth parasites to fish. Reviews in Fish Biology and Fisheries 5: 336-371.

Menni RC, RA Ringuelet \& RA Aramburu. 1984. Peces marinos de la Argentina y Uruguay, 359 pp. Editorial Hemisferio Sur, Buenos Aires.

Mundy BC \& HG Moser. 1997. Development of early stages of pelagic armorhead Pseudopentaceros wheeleri with notes on juvenile Ps. richardsoni and larval Histiopterus typus (Pisces, Percoidei, Pentacerotidae). Bulletin of Marine Science 61(2): 241-269.

Muñoz G \& V Olmos. 2008. Revisión de especies endoparásitos y hospedadoras de sistemas acuáticos de Chile. Revista de Biología Marina y Oceanografía 43(2): 173-245. 
Oliva M. 1984. Nuevos registros de tremátodos digeneos en peces marinos de Antofagasta. Ciencia y Tecnología del Mar 8: 9-15.

Parin NV \& Kotlyar. 1988. A new armorhead species, Pentaceros quinquespinis (Pentacerotidae), from the southeast Pacific. Journal of Ichthyology 28(4): 79-84.

Parin NV, GA Golovan, NP Pakhorukov, YI Sazonov \& YN Shcherbachev. 1980. Fishes from the Nazca and Sala $y$ Gomez underwater ridges collected in cruise of $\mathrm{R} / \mathrm{V}$ 'Ikhtiandr'. En: NV Parin (ed). Fishes of the open ocean, pp. 5-18. Academy of Sciences of the USSR, P.P Shirshov Institute of Oceanology, Moscow.

Paulin C, C Roberts, A Stewart \& P McMillan. 1989. New Zealand fish, a complete guide. National Museum of New Zealand, Miscellaneous Series 19: 1-279.

Pequeño G. 1989. Peces de Chile. Lista sistemática revisada y comentada. Revista de Biología Marina 24(2): 1-132.

Pequeño G. 1997. Peces de Chile. Lista sistemática revisada y comentada: Addendum. Revista de Biología Marina y Oceanografía 32(2): 77-94.

Pequeño G, A Cea-Egaña \& W Sielfeld. 1992. Primer registro en Chile para tres especies de teleósteos marinos, en base a fotografías. Boletín de la Sociedad de Biología de Concepción 63: $169-173$
Pequeño G, R Tascheri, J Merino \& P Toledo. 2003. Catálogo de la fauna íctica asociada a la pesquería del orange roughy (Hoplostethus atlanticus), 56 pp. Subsecretaría de Pesca, Instituto de Fomento Pesquero y Universidad Austral de Chile, Valparaíso.

Randall JE \& A Cea. 2010. Shore fishes of Easter Island, 154 pp. University of Hawaii Press, Honolulu.

Rego AA. 1987. Rhadinorhynchus pristis (Rudolphi, 1802) acanthocephalan parasites of fishes Scomber scombrus and $S$. japonicus. Some observations on the scanning electron microscope. Memorias do Instituto Oswaldo Cruz 82(2): 287-288.

Santos CP, DI Gibson, LER Tavares \& JL Luque. 2008. Checklist of Acanthocephala associated with the fishes of Brazil. Zootaxa 1938: 1-22.

Steindachner F. 1866. Ichthyologische Notizen (III). Über einige neue Fischarten aus Südamerika. Sitzungsberichte der Mathematisch-Naturwissenschaftlichen Classe der Kaiserlichen Akademie der Wissenschaften 53(1): 208-214.

Zama A, M Asai \& F Yasuda. 1977. Records of the pelagic armorhead, Pentaceros richardsoni, from Hachijo Island and the Ogasawara Islands. Japanese Journal of Ichthyology 24: $57-60$.

Recibido el 17 de enero de 2011 y aceptado el 16 de marzo de 2011 